\title{
EDITORIAL
}

\section{El Comité Asesor Internacional, desde el año 2009}

\author{
H umberto Reyes $\mathrm{B}^{1}$, Joaquín Palma $\mathrm{H}^{2}$, Max Andresen $\mathrm{H}^{2}$. \\ The International Advisory \\ Committee, since the year 2009
}

\begin{abstract}
Starting in 2001, Revista Médica de Chile has received advice and contributions from eight distinguished physicians from Belgium, Italy, Spain and the U.S.A., nominated Members of its International Advisory Board. A list of their contributions appears in the References section of this Editorial. The Editors have considered opportune to renew this Committee. Two of the previous members will remain in office because of their frequent and outstanding contributions. Seven new members have been selected among prominent physicians and scientists working out of Chile, with brilliant academic and professional careers, who have established regular contacts with different Chilean Societies in the fields of Internal Medicine and its subspecialties. The Editors expect to receive their advice and contributions to stimulate progress in a journal that currently has expanded its presence in the international literature in Internal and General Medicine, and in related specialties and biomedical science (Rev Méd Chile 2009; 137: 593-8).
\end{abstract}

(Key words: Journalism, medical; Peer review; Periodicals; Writing).

${ }^{1}$ Editor. ${ }^{2}$ Editor Asociado.

$\mathrm{E}^{\mathrm{n}}$ el año 2001, la Revista Médica de Chile estableció un Comité Asesor Internacional con ocho prominentes médicos, científicos y docentes de Bélgica, E.E.U.U. de N.A., España e Italia ${ }^{1}$. Varios de ellos han contribuido con publicaciones originales, particularmente revisiones de temas de su especial dedicación, han opinado sobre la marcha de la Revista, han aportado ideas para perfeccionar su proceso editorial y ampliar el alcance que debemos esperar en la difusión de los manuscritos publicados en ella. Dos miembros de este Comité han contribuido con mayor frecuencia y regularidad, reflejado en los índices de la Revista $^{2-12}$.

En el año en curso, los Editores hemos considerado oportuno renovar la nómina de miembros de este Comité. Hemos solicitado la opinión de los Presidentes y miembros prominen- tes de Sociedades filiales de la Sociedad Médica de Santiago - Sociedad Chilena de Medicina Interna para reunir sus proposiciones. Finalmente, hemos decidido prolongar la permanencia de los Dres. Felipe C. Cabello y Richard V. Lee en el Comité, agregando los nombres de siete nuevos miembros que son médicos distinguidos en el ámbito académico, con una imagen científica profesional que trasciende las fronteras de los países en que ejercen la profesión, son ejemplos de superación y liderazgo profesional, han visitado nuestro país invitados por sus pares para participar en congresos científicos locales o internacionales y mantienen contactos con nuestros propios especialistas, lo que les permite tener una idea más clara sobre la situación de las investigaciones médicas y las publicaciones científicas originadas en nuestro ambiente. De cada uno se obtuvo su anuencia 
para integrarse al Comité y el compromiso de colaboración que ello implica.

Los Editores deseamos expresar nuestro agradecimiento a los miembros que han participado generosamente desde 2001 hasta 2009 en nuestro Comité Internacional Asesor; sus opiniones sobre esta Revista seguirán siendo cálidamente apreciadas. Así también damos una cordial bienvenida a los distinguidos médicos y científicos que desde este número figuran como Miembros del Comité. Esperamos que nuestros lectores y los autores de

\section{REFERENCIAS}

1. Reyes H, Kauffmann R, Andresen M. El Comité Asesor Internacional (Editorial). Rev Méd Chile 2001; 129; 7-8.

2. Tombazzi CR, Ruely CA. Liver disease in cystic fibrosis. Rev Méd Chile 2001; 129: 1071-8.

3. LEE RV. Nausea and vomiting of pregnancy: An evolutionary hypothesis. Rev Méd Chile 2002; 130: 580-4.

4. Fernández Checa JC, Belentani S, Tiribeul C. Alcoholinduced liver disease: From molecular damage to treatment. Rev Méd Chile 2002; 130: 681-90.

5. САВELO FC. Antibióticos y acuicultura en Chile: consecuencias para la salud humana y animal. Rev Méd Chile 2004; 132: 1001-6.

6. Hernández CG, Ulloa JP, Vergara JaO, Espejo RT, САВЕLO FC. Infecciones de Vibrio parahemolyticus e intoxicaciones por algas: problemas emergentes de salud pública en Chile. Rev Méd Chile 2005; 133: 1081-8. manuscritos apreciarán la relevancia que tiene contar con ellos como un apoyo internacional para una Revista que debe cumplir su misión considerando prioritariamente los intereses de aquella proporción mayoritaria de lectores que se han autodefinido "médicos clínicos que dedican la mayor proporción de su jornada a asistencia médica, combinada con docencia o investigación y cuyas preferencias de lectura coinciden en revistas clasificadas en el área de la Medicina Interna y General"13.

7. Lee RV, De Swiet M. Obstetric Medicine: Interdisciplinary care for pregnancy and for women wishing to conceive. Rev Méd Chile 2006; 134: 109-14.

8. САВЕLO FC. Acuicultura y salud pública. La expansión de la difilobotriasis en Chile y en el mundo. Rev Méd Chile 2007; 135: 1064-71.

9. LeE RV. Medical journals: Old but not senescent. A tribute to Revista Médica de Chile on its $135^{\text {th }}$ Anniversary. Rev Méd Chile 2007; 135: 823-8.

10. САВешо FC. El Dr. Guillermo Contreras Da Silva, una figura señera en la microbiología y la salud pública chilena. Rev Méd Chile 2008; 136: 256-60.

11. СавеLO CC, САВELO FC. Zoonosis con reservorios silvestres: amenazas a la salud pública y a la economía. Rev Méd Chile 2008; 136: 385-93.

12. LEE RV. A letter from the United States: The romance of medicine - voyages and heroes. Rev Méd Chile 2009; 137: 438-43.

13. Bunout D, Reyes H. ¿Qué opinan sus lectores de la Revista Médica de Chile? Rev Méd Chile 2008; 136: 1163-8. 


\title{
Los Miembros del Comité Asesor Internacional de la Revista Médica de Chile, desde 2009
}

\author{
The Members of the International Advisory \\ Committee of Revista Médica de Chile, since 2009
}

Dame Marceia Contreras Arriagada, MD, FRCPath, FRCP (Edin) (London), F Med Sci, U.K.
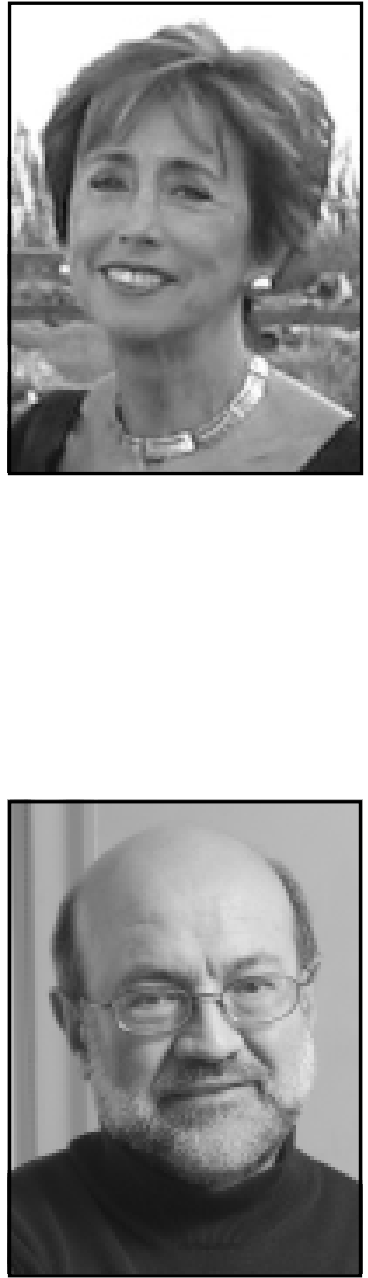

Nacida en Curicó, con infancia en Coelemu, Chile, obtuvo el título de Médico Cirujano en la Universidad de Chile (1968). Estudios de post título en Inmunología y Banco de Sangre en la Universidad de Chile. Becada en Inglaterra (1972), se radicó en ese país y se especializó en Hematología (Transfusión de Sangre) y en la organización de Bancos de Sangre, en el "National Blood Service", alcanzando el cargo de Directora. Actualmente es Profesora de Medicina Transfusional en el Royal Free Hospital Medical School, London; Profesora Visitante de la Facultad de Ciencias Aplicadas de la University of West England, Bristol, y Jefa del Programa Internacional de Transfusión Sanguínea. Por sus servicios a la salud pública en el Reino Unido, el año 2007 S.M. Isabel II le confirió el título de "Dame Commander of the British Empire." Actualmente es, además, Presidenta de la Comisión Nacional de Sangre y Tejidos, del Ministerio de Salud de Chile.

\section{Felipe C. CaBeLlo, M.D.}

Nacido en Santiago, Chile, se tituló Médico Cirujano en la Universidad de Chile (1967). Con formación de postítulo en Medicina Interna y Microbiología en la misma Universidad; desde 1972 reside en los EE.UU. de NA. Se especializó en microbiología molecular en la Universidad de Stanford. Miembro Honorario de la Academia Chilena de Medicina; Miembro Correspondiente de la Academia Chilena de Ciencias; Miembro de la Academia Americana de Microbiología y la Academia de Medicina de Nueva York. En el año 2004 recibió una beca Guggenheim en EE.UU. de NA. y Canadá. Es Profesor de Microbiología e Inmunología en el Department of Microbiology and Immunology, New York Medical College, Valhalla, NY, U.S.A. 

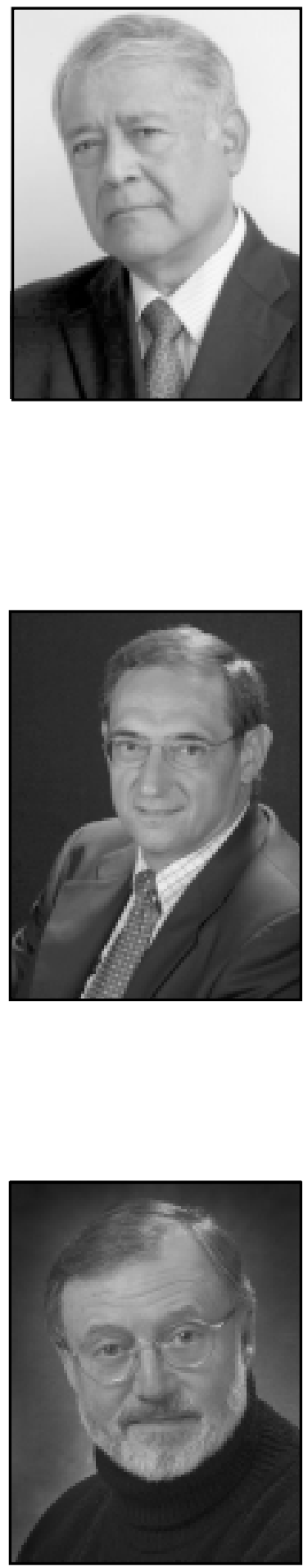

LUIS R. EsPINOZA, M.D.

Nacido en Pisco, Perú, se tituló Médico en la Universidad Cayetano Heredia, en Lima, Perú (1968). Tuvo formación de postítulo en Washington University, St. Louis, Missouri; en McGill University, Montreal, Canada; y The Rockefeller University, New York, U.S.A. Especialista en Reumatología, es Profesor de Medicina y Jefe de Reumatología en el LSU Health Sciences Center, New Orleans, U.S.A.

\section{Dr. Arturo Evangelista Masip}

Nacido en Barcelona, España. Graduado en Medicina por la Universidad Autónoma de Barcelona (1977) y Doctorado en Medicina (Cum Laude) por la misma Universidad (1992). Especialista en Cardiología, es Jefe de la Sección de Ecocardiografía y Co-Director del Departamento de Imagen Cardíaca, del Servicio de Cardiología del Hospital Universitario Vall d’Hebron, en Barcelona, España.

\section{RICHARD V. LEE, M.D.}

Born in Islip, New York State, U.S.A. Graduated from Yale College in 1960 (BS, Zoology) and from the Yale University School of Medicine with M.D. title (1964). Post graduate studies in Internal Medicine at Yale University (1964-1966 and 1969-1970). Commissioned Officer of the United States Public Health Service in the Indian Health Service at the Fort Peck Indian reservation in Montana (1966-1968) and general practitioner in Chester, Montana (1968-1969). Trained in infectious diseases, immunology and rheumatology at Yale University (1970-1972), joined its Faculty to organize the general medicine, outpatient, and medical emergency services (1972-1976). Recruited in 1976 as Professor of Medicine at 
the Medical School of the State University of New York, in Buffalo, where he is now Professor of Medicine, Pediatrics and Obstetrics, and Adjunct Professor of Anthropology and Social and Preventive Medicine. His professional and academic interests have been focused on environmental determinants for the health status of isolated, remote human populations, with field research in Chile, Brazil, Kenya, and the Himalaya Mountain Region.

\section{Dr. JULIÁN PANÉS}

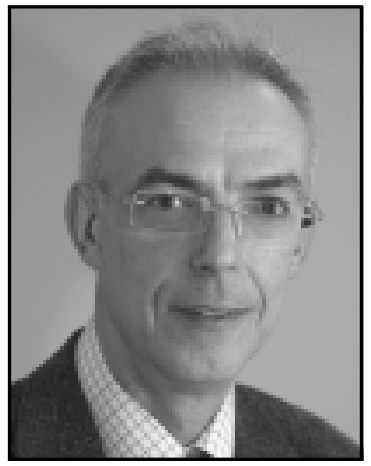

Nacido en Terrassa, Barcelona, se tituló en Medicina y Cirugía en la Universidad de Barcelona (1980) y Doctorado en Medicina en la misma Universidad (1989). Tuvo formación de postgrado en el Department of Physiology LSU, Schrevenport, LA, U.S.A. Especialista en Gastroenterología, dedicado particularmente al campo de las enfermedades inflamatorias intestinales. Es Profesor Asociado de Medicina en la Universidad de Barcelona, Jefe de la Unidad de Enfermedades Inflamatorias Intestinales y Consultor Sénior en Gastroenterología, en el Hospital Clinic de Barcelona, España.

\section{Jorge RaKela BRÖDneR, M.D.}

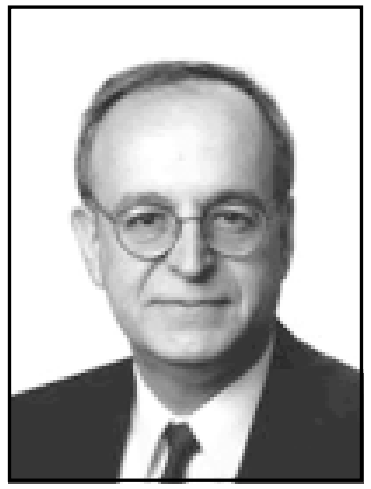

Nacido en Santiago de Chile, se tituló Médico Cirujano en la Universidad de Chile (1971). Con formación de postítulo en Medicina Interna y Virología, en la misma Universidad; desde 1974 reside en los EE.UU. de NA. Se especializó en Hepatología, desempeñándose sucesivamente en la University of Southern California en Los Angeles; en la Mayo Clinic en Rochester, Minnesota; en la Universidad de Pittsburgh y nuevamente en la Mayo Clinic, en su sede de Arizona. Miembro Honorario de la Academia Chilena de Medicina. Es Profesor de Medicina del Mayo Clinic College of Medicine y Jefe del Departamento de Medicina de la Mayo Clinic Arizona, en Scottsdale, Arizona. 


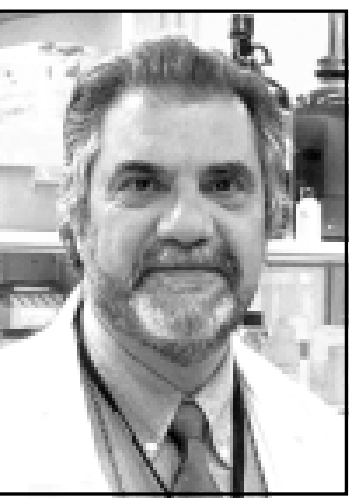

Dr. Moisés Selman Lama

Nacido en Concepción, Chile, se tituló Médico Cirujano en la Universidad de Chile (1970). Se radicó en México en 1974, cursando estudios de Neumología Médica y Biología Molecular en la Universidad Nacional Autónoma de México, con carrera de investigador y docente en el Instituto Nacional de Enfermedades Respiratorias, de la Secretaría de Salud, siendo su actual Director de Investigación. En el año 2008 recibió el Premio Nacional de Ciencias y Artes, de México, en la categoría Ciencias Físico Matemáticas y Naturales, por su destacada investigación en enfermedades pulmonares, particularmente en fibrosis idiopática.

VinCENZA T. SNOw, M.D., F.A.C.P.

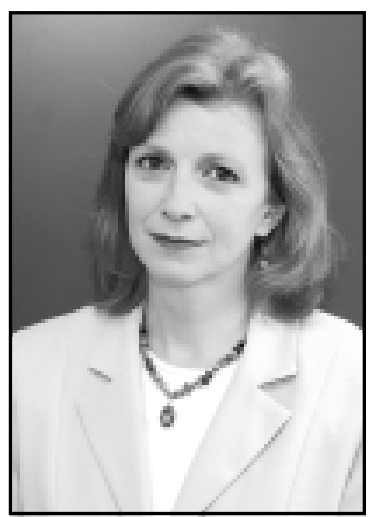

Nacida en Nueva York, EE.UU. de NA. Obtuvo "B.A. dual degree in Biological Basis of Behavior and Psychology", University of Pennsylvania, Philadelphia. Cursó estudios de medicina en la Universidad Complutense de Madrid, España, que completó en la Universidad de Chile, recibiendo el título de Médico Cirujano (1993). Formación de postítulo en Medicina Interna en Medical College of Pennsylvania, Philadelphia, se asoció al American College of Physicians con dedicación al estudio de la asistencia médica y la evaluación de la eficiencia y calidad de la atención clínica. Es Directora del "Clinical Programs and Quality of Care" del American College of Physicians, en Philadelphia, Pennsylvania, U.S.A. 